\title{
Formules empiriques pour l'étalonnage des moulinets
}

\section{Empirical rating formulae for current meters}

\author{
P.M S. IRMAY $)^{\circ}$
}

\begin{abstract}
Technique dietalonnage des monlinels; élaborution du graphique $v(n)$. .... Diverses formules 'mpirigues proposeses pont l'interpletation de ce graphique: leurs inconoinients. -- Propostlion diune nombelle formule de la forme $v=A n+[B / n+1)] ;$ inlaul de A et B par lil melhode des moindres carres; calcul simplific lorsque, au-dessus d'une certaine nilesse, $v / v$ est pratiquement constant. - l'ricisian obtenne.
\end{abstract}

Technique for calibrating current-meters; plotling of the graph v(n), Vtatous anpirical form mulae for the interpretation of his graph;

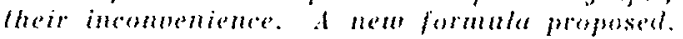

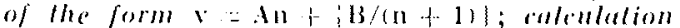
of A and li by the melhod of least squares: simplified atculalion mhen, abol' a certain

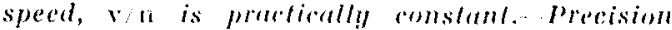
oblained.

\section{I. - INTRODUCTION}

L'étalonnage des moulinets et l'élaboration d'une formule empirique pour chaque appareil sont des opérations fort laborieuses. Ise procédé cassique consiste a tires le monlinet, a vitesse constante, le long dun canal de larage pendanl un temps ' $\mathrm{T}$, correspondant à un nombre donne $N$ de tours de l'axe de l'hélice ou des pales, et à mesurer la longueur parcourue $L$. Ia vilesse moyenne $y=\mathrm{L} / \mathrm{T}$ et le nombre moyen de lours par seconde $n=\mathrm{N} / \mathrm{T}$ dépendent de 'T, alor's que leur rapport $a=v / n=I / N$ est indépendanl du temps. Le rapport a a les dimensions d'une lonsueur. On peut done le déterminer de facon tris precise en mesurant seulement $\mathrm{L}$.

Si, sur un graphique, on porte, pour chaque valeur de $b$ et de $a$, la valeur correspondante de
Rating current melers and deriving empirical formulate for cach meter is very laborious. The usual procedure consists in dragging it al constant speed along a lowing lank for a lime $T$, which corresponds to a given number $N$ of revolutions of the axis of screw or values. The corresponding towing path 1 , is meastured. The mean velocily $v=-\mathrm{L} / \mathrm{T}$ and the mean number of revolutions per second $n=\mathrm{N} / \mathrm{T}$ depend on ' $\mathrm{l}$, whereas their ratio $a=0, n=\mathrm{I} / \mathrm{A}$ is independent of ' $T$. The ratio a has the dimension of lengith. Hance a may be delemined very arentrately by measuring $i$, only.

Plotting $b$ and a versus $n$ (fig. 1), the points

- Associale Professor. Head, Division of Hydraulic Engincering, Isracl, lustitule of Technology, Habla; Chaidman Ilydrology Section, Ismed Inion for Geodesy and Geoplyyics. 
$n$ (lig. 1), les points se réparlissent en deux zones. Pour les plus grandes vitesses (zone II), on a it pell pris cxactement :

$$
\text { b) }=\mathrm{A}^{\prime} n ; \quad \boldsymbol{t}=\mathrm{A}^{\prime}
$$

b est representéc far une droite passant par lorigine, el a a une valeur constante $A^{\prime}$. Pour des vilesses plus labbles (zono 1$)$, le frotlement fait remonler les deux courbes. Cost ainsi que pour $n=0$. $v=p_{n}$ at non 0 .

()n a alors :

$$
a \rightarrow \infty
$$

\section{II. -.- FORMULES EMPIRIQUES}

On a proposé de nombreuses formules empiriques pour la zone 1 , formules comportant deux bul trois paramitres, lelles que :

$$
\begin{aligned}
& n=A n+B \\
& n=A n+C / n \\
& b=A n+B / C / n \\
& n=A n+\sqrt{ }=A+n^{2}+C
\end{aligned}
$$

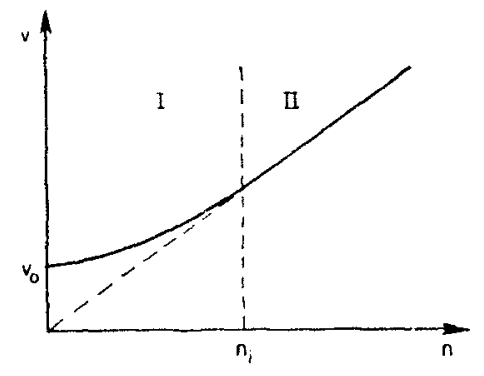

Ii1G. 1

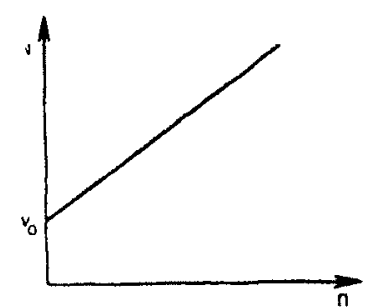

Fir. $2 a$
La premiere de ces formules est la plus simple : elle correspond à une droite avec $\mathrm{B}=v_{\|}$ fíg. 2a). Hlle ne se raceorde pas rapidement a (1), à moins que B soit très petit.

La saconde formule correspond à une hyperbole (fig. 2b) ayant pour asymptotes l'axe des " d'une part, el la droite $v=A n$, d'autre part. Cafle formule n'est pas bonne, car pour $n=0$, $n \rightarrow-$ e el pour $n_{m}=\sqrt{ } \mathrm{C} / \mathrm{A}, v$ passe par un minimum $b_{m}=2 \vee \overline{A C}$. La troisieme formule n'est gu'une variante de la deuxiome et conduit aux mimes restrictions.

Lat fuatrieme formule correspond a une hyperbole ayant pour asymptote la droite $v=-(A+B) n$. Pour $n=0, n_{0}=\overline{V C}$, et $d b / d n==A$. Cette formule est employéc par les hydrologues européns al russes, et hien que les paramètres A, $B$, C soient difficiles it calculer, elle est prilérable. lie within two zones. Al higher velocities zone II very approximately :

$$
v=A^{\prime} n ; a=A^{\prime}
$$

$n$ is a slraight line through the origin, while a has a constanl value $A^{\prime}$. Al lower velocities (zone l) friction curves both lines upwards. Thus lor $n-0, b=v_{0}$, and not zero. Here : $a \rightarrow \infty$.

\section{II.-EMPIRICAL FORMULAE}

Several empirical formulae have heen proposed for zone I, depending on 1 or 3 parameters, such as :

$$
\begin{aligned}
& n=\mathrm{A} n+\mathrm{B} \\
& n=\mathrm{A} n+\mathrm{C} / n \\
& n=\mathrm{A} n+\mathrm{B}-\mathrm{C} / \mathrm{n} \\
& n=\mathrm{A} n+\sqrt{\mathrm{B}-n^{2}-} \mathrm{C}
\end{aligned}
$$

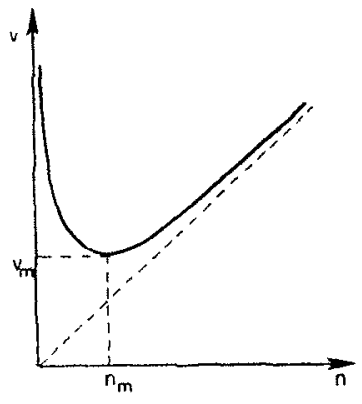

FiG. $2 b$

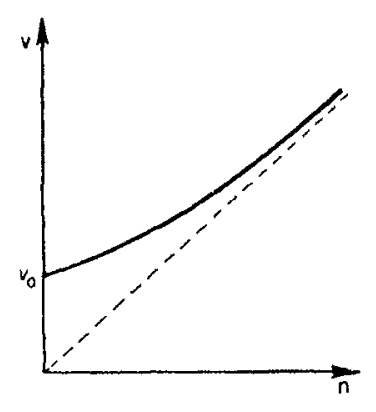

Fig. 2 r
The first formula is the simplest, as it represents a straight line with $B=v_{0}$ (fig. $2 a$ ). It does not tend rapidly towards (1), unless $B$ is very small.

The second formula is a hyperbola (fig. 2 b) wilh the $v$-axis as one asymptote and the straight line $v=A n$ as another asymptote. This formula is not good, as for $n=0, v \rightarrow \infty$ and it has for $n_{m}=\sqrt{\mathrm{C} / \mathrm{A}}$ a minimum value $b_{m}=2 \sqrt{\mathrm{AC}}$.

The lhird formula is merely a modification of the second and subject to the same limitations.

The fourth formula represents a hyperbola with the asymptote $n=(\mathrm{A}+\mathrm{B}) \boldsymbol{n}$.

$$
\text { For } n=0, n_{1}=\ldots \text { C. },(b) / d n=\mathrm{A} \text {. }
$$

II is used by European and Russian hydrologists, and though the parameters $A, B, C$ are difficult to compute, it is preferable. 


\section{III. - NOUVELLE FORMULE EMPIRIQUE}

Au Laboratoire d'Hydraulique de l'Institul de Technologie d'Isracl, lauteur s'est liver, au cours des dix derniores annés, à de nombreux essatis portant sur des moulinels WaTrs a compelle, sur de pelits moulinels OTr el sur des moulinets à hélice réalisés en Israël; ces essais l'amènent à proposer une nouvelle formule empirique simple qui concorde de très près avec les valeurs mesurées de $b$ et de $n$, et qui s'avere géneralement valable à la fois dans les zones I ef II.

$$
\begin{aligned}
& v=\mathrm{A} n+\mathrm{B} /(n+1) \\
& u=v / n=\mathrm{A}+\mathrm{b} \mathrm{B} ; \quad b=1 /[n(n+1)]
\end{aligned}
$$

B est en général beaucoup plus pelit que A. On a une hyperbole (fig. 2 (c) dont l'unc des asymptoles est la droite $v=\mathrm{A} n(n \rightarrow \infty)$, l'atute asymptote el le minimum de $v$ se trouvant dans la région de $n<0$. Quand le lemps est mesuré en secondes, $B=b_{0}$ (B est en $m / s^{2}$ ). Les paramitres $A$ of $B$ sont détermincs par la méthode des moindres carrés. Le calcul se conduit de la facon suivante : on dresse un tableau dont les colomnes donnent successivement: le numéro d'ordre de l'essai, T, I, $\mathrm{N}, a=\mathrm{I}, \mathrm{N}, n=\mathrm{N} / \mathrm{T}, b=1 /[n(n+1)]$, $b: a b, v=\mathrm{L} / \mathrm{T}$, et la valeur de $v$ calculé ensuite d'apris la formule (3).

Puis on lait la somme des chillies des colonnes $a, b, b^{\text {? }}, a b$.

La méthode des moindres carrés consiste à rechercher le minimum de la fonction.

$$
\mathrm{F}(\mathrm{A}, \mathrm{B})=\mathrm{N}\left(\mathrm{A}+\mathrm{b} \mathrm{B}-(a)^{2}\right.
$$

ce minimum est atteint lorsqu'on a simultancment :

$$
\frac{\partial \mathrm{F}}{\partial \mathrm{A}}=0 \quad \frac{\partial \mathrm{F}}{\partial \mathrm{B}}=0
$$

oll :

$$
\begin{gathered}
k A+B . \Sigma b=\Sigma a \\
A \cdot \Sigma b+B . \Sigma b^{2}= \pm a b
\end{gathered}
$$

aì $k$ est le nombre des points d'essais considéris comme valables. La solution est :

$$
\mathrm{A}=\mathrm{D} \mathrm{N}_{\mathrm{N}} / \mathrm{D} ; \quad \mathrm{B}=\mathrm{I} \mathrm{D}_{\mathrm{n}} / \mathrm{D}
$$

avec:

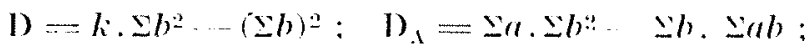

$$
\begin{aligned}
& 1)_{13}=k . \Sigma a b-\Sigma a . \Sigma b
\end{aligned}
$$

Les equations (3) permellent alors de calculer les valeurs de $b$, qui sont comparés avec les valeurs experimentales $v=\mathrm{I} / \mathrm{T}$.

\section{III.-A NEW EMPIRICAL FORMULA}

Numerous tests on Wrats cup-melers, small OrTs and Israël produced serew-meters, carried out hy the author at the Hydraulics Laboratory of the Institule of 'lechnology, Haifa, during the last ten gears, suggest a new simple empirical formula which fits elosely the moasured $v, n$ values, and is usmally valid both in zomes I and II.

$$
\begin{aligned}
& v=A n+B /(n+1) \\
& a=b / n \cdots A+b B ; \quad b=1 /[n(n+1)
\end{aligned}
$$

$B$ is ustally much smaller than $A$, This is a hyberbola (fig. 2 c) asymptotic to the straght line $v=$ An for $\mathrm{n} \rightarrow \infty$, with the other asymptote and minimum value outside the zone of positive n. When the time is measured in seconds, $B=b_{0}$ (the unit of $B$ is $11 / \mathrm{s}^{2}$ ). The paramelers $A, B$ are determined by the method of least squares. The computation proceds as follows: a table is prepared, the columms of which give the ordinal number of the lest, T, $\mathrm{L}, \mathrm{N}, a=\mathrm{L} / \mathrm{N}, n=\mathrm{N} / \mathrm{T}$, $b=1 /[n(n+1)], b^{2}, a b, b=\mathrm{L} / \mathrm{T}, b$ computed later on by (3).

The sums of the columns $a, b, b-a b$ are compuled.

The method of least squares requires the minimum of the function :

$$
F(A, B)=\Sigma(A+b B, \quad a)::
$$

which is reached, when simullaneously :

$$
\begin{aligned}
& \partial \mathbf{F}=0 \quad \partial \mathbf{F} \\
& \partial \mathrm{H}
\end{aligned}
$$

or :

$$
\begin{gathered}
k A+B . \Sigma b=\Sigma a \\
\text { A. } \Sigma b+B . \Sigma b \div=\Sigma a b
\end{gathered}
$$

where $l$ is the number of ralid lest points. 'The solulion is :

$$
\left.\left.A=1)_{.} /(1) ; \quad B \ldots 1\right)_{1} / 1\right)
$$

where:

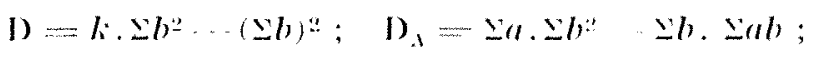

$$
D_{1}=k . \check{a} b b \ldots \mathrm{\Sigma} a, \Sigma b
$$

Then (3) gives the emmputed values of 1 ; which are then compared with the axperimental $v=I / \mathrm{T}$. 


\section{IV. - CALCUL SIMPLIFIÉ}

On peut tris sourent simplifier les calculs, lorsque l'examen du lableau des résultats montre que a est pratiquement constant pour les plus grandes vitesses, par exemple pour $n>n_{i}$, soit dans la zone II de la figure 1 , ou la formule (1) est valable. Dans ce cas :

$$
\begin{aligned}
& \Lambda^{\prime}=\left(\frac{k}{i} a\right) /\left(k \text { i) pour } n>n_{i}\right. \\
& \text { (ou } n \geqslant n^{\prime} \# n_{i} \text { ) }
\end{aligned}
$$

Si, par exemple, $n_{i}=0,621$, nous arrondirons ce chiffre it $\mathrm{n}^{\prime}=0,600$, afin de simplifier les calculs. Dans la zone 1 , nous prendrons de nouvaau les formules (3). En un point queleonque $n$ nous arons :

$$
a=\mathrm{A}+b \mathrm{~B} ; \quad b=1 /[n(n+1)]
$$

Au point donne $n=n^{\prime}$, nous avons :

$$
a^{\prime}=A+b^{\prime} B ; \quad b^{\prime}=1 /\left[n^{\prime}\left(n^{\prime}+1\right)\right]
$$

oll, par soustraction :

$$
\mathrm{B}\left(b \ldots b^{\prime}\right)=a+\cdots a^{\prime}
$$

Suivant la méthode des moindres anres, le minimum de la fonction:

$$
f(B)-\Sigma\left[B\left(b \cdots b^{\prime}\right) \ldots\left(a \ldots\left(a^{\prime}\right)\right]^{2}\right.
$$

ast alleint lorsque $d / / d B=0$, ou :

$$
\left\{\begin{array}{l}
B=\geq\left(a \cdots a^{\prime}\right)\left(b \ldots b^{\prime}\right) / \triangle\left(b-\ldots b^{\prime}\right) 2 \\
A=a^{\prime} \ldots b^{\prime} B
\end{array}\right.
$$

Le tableau est en consciquence simplific pour $n \leqslant n^{\prime}$ :

n" d'ordice de l'essai, T, L, N, $a=\mathrm{L} / \mathrm{N}, a-a^{\prime}$, $n=\mathrm{N} / \mathrm{T}, \quad b=1 /[n(n+1)], \quad b-b^{\prime}, \quad\left(b-b^{\prime}\right)^{*}$, $\left(a-a^{\prime}\right)\left(b-b^{\prime}\right), b=\mathrm{L} / \mathrm{T}, v$ calculé d'après $(3)$

Le tableau précedent peul d'ailleurs atre employe, car :

$$
\begin{aligned}
& \Sigma\left(b-\ldots b^{\prime}\right)^{2}=\Sigma b^{2}-2 b^{\prime} \leq b+(k \cdots i) b^{\prime-2} \\
& \Sigma\left(a \cdots a^{\prime}\right)\left(b-b^{\prime}\right)=\Sigma a b \\
& +(k-i) a^{\prime} b^{\prime}-b^{\prime} \Sigma a \ldots a^{\prime} \pm b
\end{aligned}
$$

\section{V. - PRECISION}

I. est généralement mesuré à $\pm 1 \mathrm{~cm}$ près : done, $\Delta I_{1}=0,01 \mathrm{~m}$. En utilisant un chronometre

\section{IV.-SIMPLIFIED COMPUTATION}

Very often this computation may be simplified, when the inspection of the table shows that a is practically constant for the higher velocities, e.g. for $n>n_{i}$, zone II of lig. 1 , for which (1) is valid.

Here :

$$
\begin{aligned}
A^{\prime}=\left(\begin{array}{l}
k \\
\stackrel{i}{i} a
\end{array}\right) /(k \cdots-i) \text { for } n>n_{i} \\
\text { (or } \left.n \geqslant n^{\prime} \# \mathbf{n}_{i}\right)
\end{aligned}
$$

If e.s. $n_{i}=0.621$, we shall round it ofl lo $n^{\prime}=0.600$, in order to simplify the compulalions. In zone 1 we shall again assume (3). Al any poinl $n$ we have :

$$
a=A+b \mathrm{~B} ; \quad b=1 /[n(n+1)]
$$

At he given point $n=n^{\prime}$ we have:

$$
a^{\prime}=\mathrm{A}+b^{\prime} \mathrm{B} ; \quad b^{\prime}=1 /\left(n^{\prime}\left(n^{\prime}+1\right)\right]
$$

By substraction :

$$
\mathrm{B}\left(b \cdots b^{\prime}\right)=a \cdots a^{\prime}
$$

By the method of leasl spuares the minimum of the function:

$$
f(B)=\Sigma\left(B\left(b-\ldots b^{\prime}\right) \ldots\left(a-\ldots a^{\prime}\right)\right]^{ \pm}
$$

is rached when $d /$; $d B=0$, or:

$$
\left\{\begin{array}{l}
\mathrm{B}=\mathrm{z}\left(a \ldots a^{\prime}\right)\left(b \ldots b^{\prime}\right) / \mathrm{a}\left(b \ldots b^{\prime}\right)^{2} \\
\mathrm{~A}=a^{\prime} \ldots b^{\prime} \mathrm{B}
\end{array}\right.
$$

The table is accordingly simplified for $n \leqslant n^{\prime}$ : ()'dinal number of the test, 'T, L, N, $a=\mathrm{I} / \mathrm{N}$, $a-a^{\prime}, n=\mathrm{N} / \mathrm{T}, b=1 /[n(n+1)], b-b^{\prime},\left(b-b^{\prime}\right)^{2}$, $\left(a-a^{\prime}\right)\left(b-b^{\prime}\right), v=\mathrm{L} / \mathrm{T}, b_{\text {romp }}$ by $(3)$.

The provious table may also be used, as :

$$
\begin{aligned}
& \Sigma\left(b \ldots-b^{\prime}\right)^{2}=\Sigma b^{\prime}-2 b^{\prime} \leq b+(k-\ldots i) b^{\prime 2} \\
& \Xi\left(a-\ldots-a^{\prime}\right)\left(b-b^{\prime}\right)=\Sigma a b \\
& +(k-i) a^{\prime} b^{\prime} \ldots-b^{\prime} \pm a-a^{\prime} \geq b
\end{aligned}
$$

\section{V.-PRECISION}

Usually $L$ is measured within $\pm 1 \mathrm{~cm}$, so \lrcorner $\mathrm{I}=0.01 \mathrm{~m}$. When a stop watch is used 
$\Delta \mathrm{T}=0,1 \mathrm{~s}$. Par conséquent, l'erreur absolue sur $a$ est tris petite : $\Delta a=\Delta \mathrm{I} / \mathrm{N}=0,01 / \mathrm{N}$. lo nombre de tours $N$ est voisin de 100 , el tres rarement inférieur à 50 . " variant généralement entre 0,1 et 1 (système métrique), a est connu à moins de $0,1 \%$ près.

Les erreurs relatives sur $v$ el $n$ son beaucoup' plus élevées.

$$
\Delta v / v=\Delta \mathrm{L} / \mathrm{L}+\Delta \mathrm{T} \mathrm{T}=0,01 / \mathrm{L}+0,1 / \mathrm{T}
$$

En genéral, $\mathrm{T}>10 \mathrm{~s}, \mathrm{I}>20 \mathrm{~m}$; par consequent, pratiquement : $\lrcorner v / v=0,1 \mathrm{~T}$, soil à peu pres 0,1 à $1 \%$.

$$
\Delta n / n=\Delta \mathrm{T} / \mathrm{T}=0,1 / \mathrm{T}
$$

igalenuent à peu près 0,1 à $1 \%$.

La précision de la formule (3) dépend principalement de $A$, car $B$ est très petit. Or, par suite de (4), (5), A est très sensihle aux plus frandes valeurs de $b^{*}$, ou aux petites valeurs de n. Il est recommandable de ne pas tenir compte, dans les sommes, des valeurs de $n$ vaiment tris petiles, afin d'éviter une exagération de celle influence. $\perp \mathrm{T}=0.1$ s. Hence the absolute error on $a$ is very small $\Delta a=\Delta \mathrm{L} / \mathrm{N}=0.01 / \mathrm{N}$.

The number of revolutions $\mathrm{N}-100$, very seldom less than 50 . As $a$ varies usually between 0.1 and 1 (metric units), $a$ is known within $0.1 \%$.

The relative errors on $v$ and $n$ are considerably higher :

$$
\Delta n / v=\Delta \mathrm{L} / \mathrm{L}+\Delta \mathrm{T} \mathrm{T}=0.01 / \mathrm{I}+0.1 \mathrm{~T}
$$

Usually $\mathrm{T}>10 \mathrm{~s}, \mathrm{~L}>20 \mathrm{~m}$; hence pratically $\Delta v / v=0.1 \mathrm{~T}$, or of the order of 0.1 to $1 \%$

$$
\lrcorner n / n= \pm \mathrm{T} / \mathrm{T}=0.1 / \mathrm{T}
$$

also of the order of 0.1 lo 1 \%

The precision of the formula (3) depends essentially on $A$, as $B$ is very small. Or, hecause of (4), (5), A is much alfected hy the higher values of $b^{2}$, of small values of $n$. It is advisable fo reject from the sums the very smallest values of $n$, so as to avoid this unwaramted exaggerated alfect.

\section{ERRATUM}

La Howille Blanche, n" 3/1954, p. 379 :

1" Fig. 2. - Les ordonnées sont évidemment : $0,400,0,5100 \ldots$ et non $400,500 \ldots$

2" Jegende de la figure 4. Il faut lire:

\section{Lissuis ale Donges}

Variation du coefficient $;$ en fonction du nombre de Reynolds. (les resultats d'essais américains sont extraits de la figure 1, page 594 de la Howile Blanche, aout-septembre 1952 , elle-mème extraite de la figure 19, page 1067, volume 113, A.S.C.E., 1948. Pour l'équation de Biesel, cf. la Houille Blanche, n" A, 1954, page 255.) 